\title{
Vagus nerve electrical stimulation inhibits serum levels of S100A8 protein in septic shock rats
}

\author{
MING LEI ${ }^{1}$ and XIN-XIN LIU ${ }^{2}$ \\ ${ }^{1}$ Department of Critical Care Medicine, Shanghai Seventh People's Hospital, Shanghai 200137; \\ ${ }^{2}$ Shanghai Institute of Medical Genetics, Shanghai Children's Hospital Affiliated to Shanghai Jiao-Tong University, \\ Shanghai 200040, P.R. China
}

Received March 15, 2015; Accepted February 18, 2016

DOI: $10.3892 / \mathrm{mmr} .2016 .5002$

\begin{abstract}
The vagus nerve and the released acetylcholine exert anti-inflammatory effects and inhibit septic shock. However, their detailed mechanisms remain to be elucidated. The present study aimed to investigate the effects of vagus nerve electrical stimulation on serum S100A8 levels in septic shock rats. A total of 36 male Sprague-Dawley rats were randomly divided into six equal groups: i) Sham group, receiving sham operation; ii) CLP group, subjected to cecal ligation and puncture (CLP) to establish a model of polymicrobial sepsis; iii) VGX group, subjected to CLP and bilateral cervical vagotomy; iv) STM group, subjected to CLP, bilateral cervical vagotomy and electrical stimulation on the left vagus nerve trunk; v) $\alpha$-bungarotoxin (BGT) group was administered $\alpha$-BGT prior to electrical stimulation; vi) Anti-receptor for advanced glycation end products (RAGE) group, administered intraperitoneal injection of anti-RAGE antibody prior to electrical stimulation. The right carotid artery was cannulated to monitor mean artery pressure (MAP). The serum aspartate aminotransferase (AST) and alanine aminotransferase (ALT) levels were measured to assess the liver function. Serum S100A8 and advanced glycation end product (AGE) levels were measured using enzyme-linked immunosorbent assays. The expression of hepatic RAGE was determined by western blotting. The present study revealed that Sprague-Dawley rats exhibited progressive hypotension and significantly increased serum AST and ALT levels following CLP challenge compared with the sham group. The levels of S100A8 and AGEs, and the protein expression of hepatic RAGE were significantly increased following CLP compared with the sham group. Vagus nerve electrical stimulation significantly
\end{abstract}

Correspondence to: Dr Xin-Xin Liu, Shanghai Institute of Medical Genetics, Shanghai Children's Hospital Affiliated to Shanghai Jiao-Tong University, 1400 West Beijing Road, Shanghai 200040, P.R. China

E-mail: liuxxsh@126.com

Key words: vagus nerve, electrical stimulation, septic shock, cecal ligation and puncture, S100A8 prevented the development of CLP-induced hypotension, alleviated the hepatic damage, reduced serum S100A8 and AGEs production, and reduced the expression of hepatic RAGE. The inhibitory effect of vagus nerve electrical stimulation was reversed by pre-treatment with $\alpha$-BGT and enhanced by pre-treatment with anti-RAGE antibody. In conclusion, vagus nerve electrical stimulation may produce a protective effect on septic shock in rats by decreasing the production of S100A8.

\section{Introduction}

Septic shock is a complex pathophysiological process characterized by hypotension, systemic inflammatory response syndrome (SIRS) and widespread multiple organ dysfunction syndrome (MODS), with a high mortality (1). At present, a variety of pro-inflammatory cytokines are excessively produced in the development of hypotension, tissue damage and organ injury during sepsis/septic shock $(2,3)$.

S100A8 is a member of S100 calcium-binding family of proteins, and can combine with its binding partner S100A9 to form a heterodimer of S100A8/A9 dimers (4). S100A8 protein is constitutively expressed in neutrophils and can be induced in monocytes and endothelial cells (5). S100A8 serves a variety of immune functions, including antibacterial activity, cell death induction, pro- and anti-inflammatory properties, and activation of endothelial cells (6-9). The levels of S100A8 have been shown to be increased following septic shock and decreased along recovery at both the protein and mRNA expression levels (10).

In recent years, the vagus nerve and the release of neurotransmitters acetylcholine demonstrate anti-inflammatory effect and reduce the production of pro-inflammatory cytokines and lethal effect of biological toxin (11-13). Stimulation of the vagus nerve has potential protective effects on the damaging effects of cytokine release, including endotoxemia, sepsis, ischemia/reperfusion injury, arthritis and other inflammatory syndromes (14). Furthermore, vagus nerve stimulation demonstrated protective roles against septic shock in rats (15). However, the precise mechanisms underlying the anti-inflammatory effects of the vagus nerve remain to be elucidated.

The present study established a septic shock model in rats by cecal ligation and puncture (CLP), a model of polymicrobial sepsis, and aimed to investigate the effects of vagus nerve 
electrical stimulation on hemodynamics, liver function, the serum level of S100A8 and advanced glycation end products (AGEs), and the protein expression of hepatic receptor for advanced glycation end products (RAGE) following CLP challenge. $\alpha$-bungarotoxin ( $\alpha$-BGT), an antagonist against nicotinic acetylcholine receptor $\alpha 7$ ( $\alpha 7-\mathrm{nAChR})$ subunit, and anti-RAGE antibody were applied in combination with vagus nerve electrical stimulation to examine the precise target point of the vagus nerve on septic shock and S100A8.

\section{Materials and methods}

CLP model. Male Sprague-Dawley rats (SLRC Laboratory Animal Centre, Shanghai, China) weighing 200-250 g were used to establish a polymicrobial sepsis model by the CLP procedure, which was performed, as described previously (16). Briefly, the animals were anesthetized with an intraperitoneal injection of ketamine $(80 \mathrm{mg} / \mathrm{kg}$; Henry Pharaceutical Co., Ltd., Jiangsu, China) and xylazine (12 mg/kg; Sigma-Aldrich, St. Louis, MO, USA). A $2 \mathrm{~cm}$ incision was made near the lower midline of the abdomen to isolate the cecum. To ligate $\sim 30 \%$ of the cecum just below the ileocecal valve, to avoid bowel obstruction, 3.0 silk was used. This selected percentage of cecum ligation revealed a reproducible rate of mortality in Sprague-Dawley rats (17). An $18 \mathrm{~g}$ needle was used to puncture twice the ligated portion of cecum, causing a small quantity of contents expel into the abdominal cavity. Following this, the cecum was returned to its original location and the abdominal incision was closed in muscle and skin layers. No antibiotics were administered; however, the animals received normal saline $(20 \mathrm{ml} / \mathrm{kg})$ subcutaneously as fluid resuscitation. The present study was performed according to the protocol approved by Animal Care and Study Committee of Shanghai Children's Hospital Affiliated to Shanghai Jiao-Tong University (Shanghai, China).

Experimental protocols. A total of 36 rats were randomly divided into six equal groups: i) Sham group, receiving sham operation; ii) CLP group, subjected to CLP and the bilateral vagal trunks were isolated but not transected; iii) VGX group, subjected to CLP and bilateral cervical vagotomy; iv) STM group, subjected to CLP and bilateral cervical vagotomy, followed by bipolar platinum electrodes being connected to the left vagus nerve trunk in a stimulation module and controlled by an acquisition system; v) $\alpha$-BGT group, administered $\alpha$-BGT (1.0 $\mu \mathrm{g} / \mathrm{kg}$, i.v.; Sigma-Aldrich) prior to vagus nerve electrical stimulation and following CLP and bilateral cervical vagotomy; vi) anti-RAGE group, administered with intraperitoneal injection of anti-RAGE neutralizing antibody (1 mg/kg; Beijing Bioss Biotech Co., Ltd., Beijing, China) prior to stimulation of the vagus nerve and following CLP and bilateral cervical vagotomy. The mean artery pressure (MAP) was monitored by cannulating into the right carotid artery.

Vagus nerve stimulation. The bipolar platinum electrodes were connected to left vagus nerve trunk in a stimulation module and controlled by an acquisition system. The vagus nerve was subjected to constant electrical stimulation (left cervical vagus, $5 \mathrm{~V}, 2 \mathrm{~ms}, 1 \mathrm{~Hz}$ ). Immediately following CLP, stimulation was performed for $20 \mathrm{~min}$. Firstly, the left vagus nerve trunk was exposed and isolated without transection. The animal was subsequently subjected to CLP operation, followed by cutting off the left vagus nerve trunk and electrical stimulation.

Measurement of liver function markers. The serum was separated from blood samples of Sprague-Dawley rats at $6 \mathrm{~h}$ after CLP by centrifugation (2,000 x g per min for $10 \mathrm{~min})$, and was analyzed within $24 \mathrm{~h}$ using a chemistry analyzer (Hitachi 7170; Hitachi, Tokyo, Japan). Serum levels of alanine aminotransferase (ALT) and aspartate aminotransferase (AST) were measured to assess the liver function.

Measuring serum levels of S100A8 and AGEs by enzyme-linked immunosorbent assay (ELISA). At 3 and $6 \mathrm{~h}$ after CLP or sham CLP operation, the whole blood sample was drawn via the right carotid artery ( $2 \mathrm{ml}$ each). The serum was immediately separated by centrifugation at $2,000 \mathrm{x}$ for $15 \mathrm{~min}$ at $4^{\circ} \mathrm{C}$, and was divided into aliquots and stored at $-70^{\circ} \mathrm{C}$ for assay. ELISA was performed to measure serum levels of S100A8 (Hycult Biotechnology b.v., Uden, the Netherlands) and AGEs (Xitang Bio Technology Co., Ltd., Shanghai, China), according to the manufacturer's protocol. Ortho-phosphoric acid (100 $\mu \mathrm{l})$ was added to terminate the color reaction. A microplate reader (Spectra MR; Dynex, Chantilly, VA, USA) was used to acquire data. The concentrations of S100A8 or AGEs in the samples were calculated based on standard curves.

Western blot analysis. The rat hepatic tissue was dissected for analyzing protein levels by western blotting. The proteins from hepatic tissue were extracted and their concentrations were determined by bicinchoninic acid protein concentration assay kit (Beijing Biosea Biotechnology Co., Ltd., Beijing China). The cell lysates $(50 \mu \mathrm{g})$ were separated by $10 \%$ sodium dodecyl sulfate-polyacrylamide gel electrophoresis and the proteins were subsequently electrophoretically transferred onto polyvinylidene difluoride membranes (Bio-Rad Laboratories, Inc., Hercules, CA, USA). The membrane was blocked in $5 \%$ non-fat dry milk for $60 \mathrm{~min}$ in Tris-buffer saline, containing $0.05 \%$ Tween 20 , at room temperature. The blots were incubated with horseradish peroxidase (HRP)-conjugated rabbit anti-rat antibodies against RAGE (cat. no. sc-33662; 1:1,000) or $\beta$-actin (cat. no. sc-1616; 1:1,000) for $1 \mathrm{~h}$ at $37^{\circ} \mathrm{C}$ (Santa Cruz Biotechnology, Inc., Santa Cruz, CA, USA). The protein bands were detected using enhanced chemiluminescence (Pierce ${ }^{\circledR}$ ECL Plus Western Blotting Substrate; Pierce Biotechnology, Inc., Rockford, IL, USA). Gel-Pro Analyzer software (version 4.0; Media Cybernetics, Rockville, MD, USA) was applied to analyze relative intensities of protein bands.

Statistical analysis. The data are expressed as mean \pm standard deviation. Statistical analysis was performed using commercially available software (SPSS version 14.0; SPSS, Inc., Chicago, IL, USA). A one-way analysis of variance was used to assess the differences between groups at the same time point, and two-way analysis of variance was used for repeated measurements with multiple comparisons (Bonferroni test). $\mathrm{P}<0.05)$ was considered to indicate a statistically significant difference. 


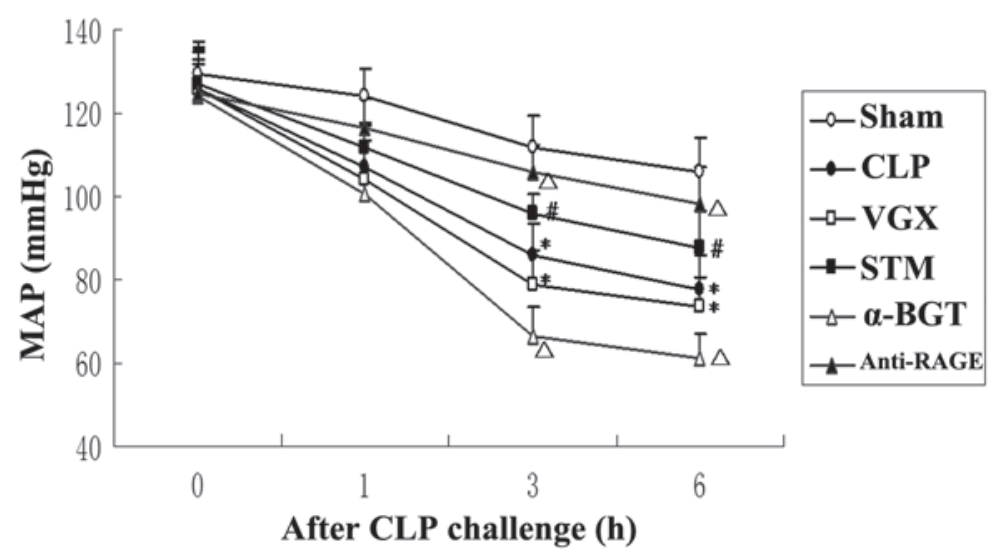

Figure 1. Changes of the MAP. The MAP following CLP challenge was assessed for the Sham, CLP, VGX, STM, $\alpha$-BGT and anti-RAGE groups. The data are expressed as the mean \pm standard deviation $\left(" \mathrm{P}<0.05\right.$, compared with the Sham group; ${ }^{*} \mathrm{P}<0.05$, compared with the CLP group; ${ }^{\Delta} \mathrm{P}<0.05$, compared with the STM group). MAP, mean artery pressure; CLP, cecal ligation and puncture; VGX, CLP + bilateral cervical vagotomy; STM, CLP + bilateral cervical vagotomy + electrical stimulation; BGT, bungarotoxin; RAGE, receptor for advanced glycation end products.

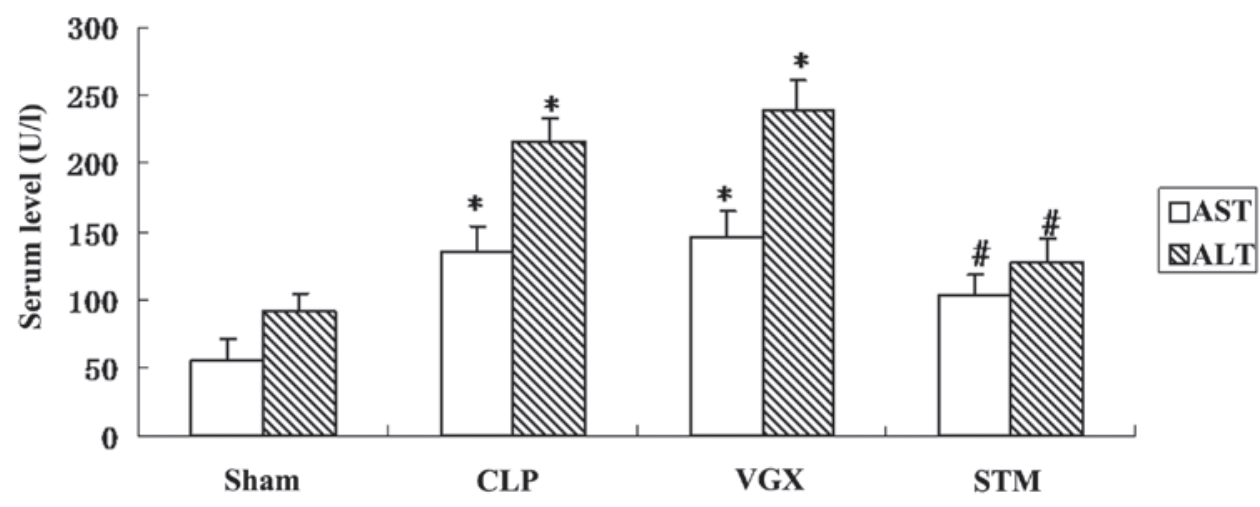

Figure 2. Serum levels of AST and ALT $6 \mathrm{~h}$ after CLP challenge ( $\mathrm{n}=6)$. The data are expressed as the mean \pm standard deviation $\left({ }^{*} \mathrm{P}<0.05\right.$, compared with the Sham group; ${ }^{\#} \mathrm{P}<0.05$, compared with the CLP group. CLP, cecal ligation and puncture; VGX, CLP + bilateral cervical vagotomy; STM, CLP + bilateral cervical vagotomy + electrical stimulation; AST, aspartate aminotransferase; AST, alanine aminotransferase.

\section{Results}

$M A P$. At baseline and $1 \mathrm{~h}$ after CLP, no significant differences were observed in the MAP between any of the groups. In the CLP group, MAP was significantly decreased compared with the sham group 3 and $6 \mathrm{~h}$ after CLP challenge $(\mathrm{P}<0.05)$. At 3 and $6 \mathrm{~h}$ after CLP challenge, the MAP increased significantly in the STM group compared with the sham group $(\mathrm{P}<0.05)$. The inhibitory effect on septic shock of vagus nerve electrical stimulation was attenuated by $\alpha$-BGT pre-treatment and enhanced by anti-RAGE antibody pre-treatment. After CLP challenge, MAP was decreased in the VGX group compared with the CLP group, with no significant difference (Fig. 1).

Blood biochemistry evaluation. To evaluate the hepatic injury by CLP induced septic shock, the serum AST and ALT levels were measured in Sprague-Dawley rats at $6 \mathrm{~h}$ after CLP. Serum AST and ALT levels were significantly increased by CLP challenge compared with the sham group $(\mathrm{P}<0.05)$. By contrast, the STM group exhibited significantly decreased serum AST or ALT levels compared with that in the CLP group ( $\mathrm{P}<0.05$; Fig. 2).
Serum levels of S100A8. To examine whether vagus nerve electrical stimulation can modulate the production of S100A8 in septic shock rats, ELISA was performed to measure serum S100A8 levels. The serum levels of S100A8 were significantly increased at 3 and $6 \mathrm{~h}$ after the CLP induction. More serum S100A8 production was observed after bilateral cervical vagotomy compared with the CLP group, with no significant difference. The left vagus nerve electrical stimulation significantly reduced serum production of S100A8. $\alpha$-BGT pre-treatment significantly reversed, while anti-RAGE antibody pre-treatment significantly enhanced the inhibitory effect of vagal electrical stimulation in serum S100A8 level (Fig. 3).

Serum levels of AGEs. The serum levels of AGEs were measure by ELISA at 3 and $6 \mathrm{~h}$ after CLP. Compared with the sham group, a significant increase in the serum levels of AGEs was observed in the CLP group $(\mathrm{P}<0.05)$. Vagus nerve electrical stimulation significantly reduced the levels of AGEs compared with the CLP group. The inhibitory effect on serum levels of AGEs was significantly reversed by $\alpha$-BGT pre-treatment and enhanced by anti-RAGE antibody pre-treatment (Fig. 4). 


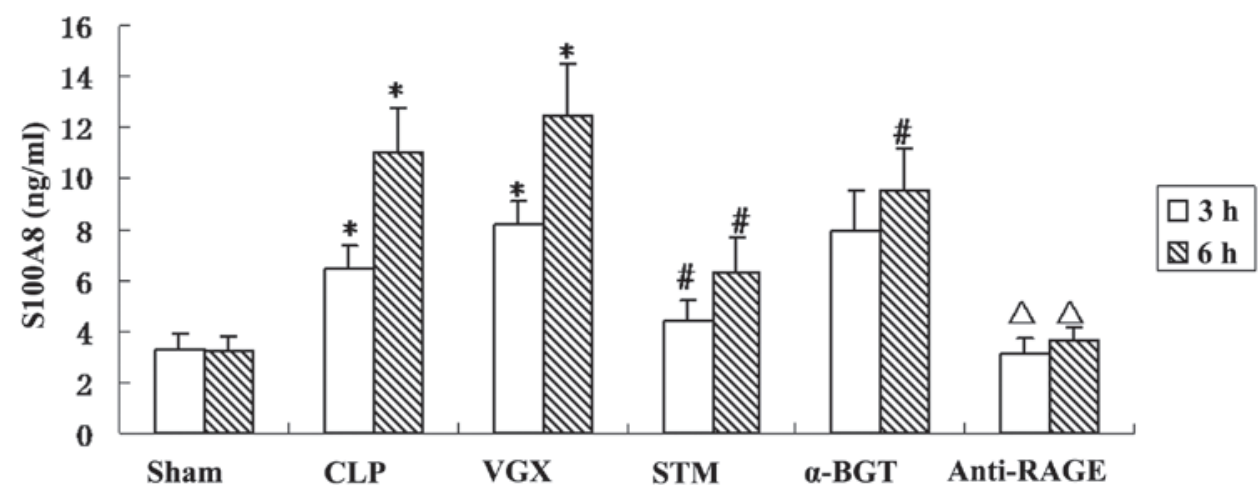

Figure 3. Serum levels of S100A8 at 3 and $6 \mathrm{~h}$ after CLP challenge $(\mathrm{n}=6)$. An enzyme-linked immunosorbent assay was performed to measure the serum levels of S100A8 in Sprague-Dawley rats. The data are expressed as the mean \pm standard deviation ( ${ }^{*} \mathrm{P}<0.05$, compared with the Sham group; $\mathrm{P}<0.05$, compared with the CLP group; ${ }^{\triangle} \mathrm{P}<0.05$, compared with the STM group). CLP, cecal ligation and puncture; VGX, CLP + bilateral cervical vagotomy; STM, CLP + bilateral cervical vagotomy + electrical stimulation; BGT, bungarotoxin; RAGE, receptor for advanced glycation end products.

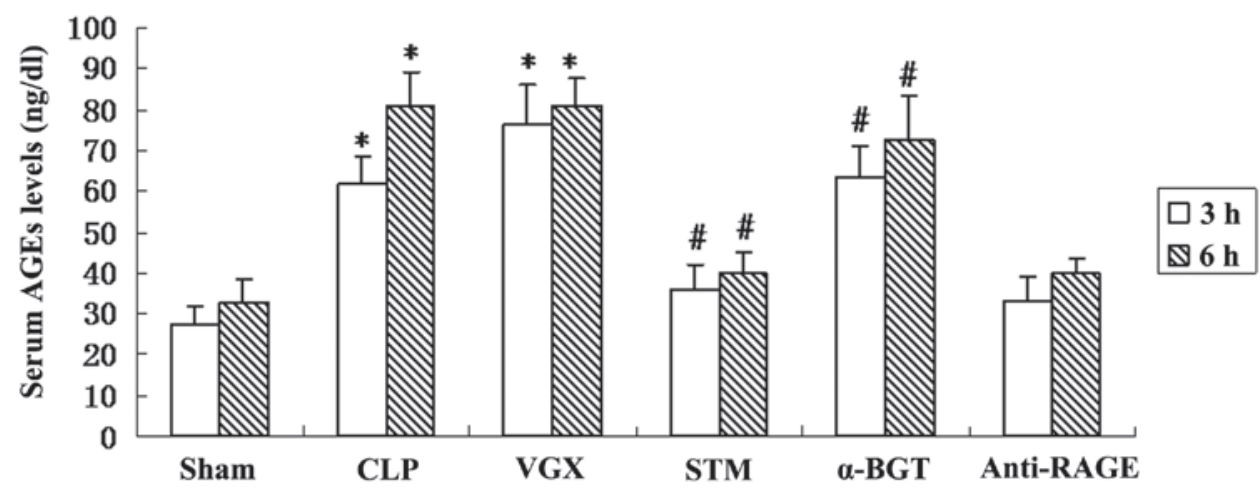

Figure 4. Serum levels of AGEs levels in different groups at 3 and $6 \mathrm{~h}$ after CLP challenge (n=6). An enzyme-linked immunosorbent assay was performed to measure the serum levels of AGEs in Sprague-Dawley rats. The data are expressed as the mean \pm standard deviation ( $\mathrm{P}<0.05$, compared with the Sham group; ${ }^{\text {"P }}<0.05$, compared with the CLP group). CLP, cecal ligation and puncture; VGX, CLP + bilateral cervical vagotomy; STM, CLP + bilateral cervical vagotomy + electrical stimulation; BGT, bungarotoxin; RAGE, receptor for advanced glycation end products; AGEs, advanced glycation end products.

Hepatic protein expression of RAGE. Western blotting was performed to detect the hepatic protein expression of RAGE at $6 \mathrm{~h}$ after CLP challenge. The protein expression of hepatic RAGE was significantly increased in the CLP group compared with the sham group $(\mathrm{P}<0.05)$. Vagus nerve electrical stimulation significantly decreased the protein expression of hepatic RAGE compared with the CLP group $(\mathrm{P}<0.05)$. By contrast, no significant differences were observed in the hepatic RAGE protein expression between the CLP and VGX groups (Fig. 5).

\section{Discussion}

Previously, the cholinergic anti-inflammatory pathway was identified to associate the central nervous system with the immune system (18). In the present study, a septic shock model was established in Sprague-Dawley rats by CLP. This revealed that CLP provoked progressive hypotension and liver damage, elevated serum S100A8 and AGEs levels, and enhanced the protein expression of hepatic RAGE. Application of electrical stimulation to the vagus nerve significantly attenuated the CLP-induced hypotension and liver damage, decreased serum S100A8 and AGEs levels, and reduced the protein expression of hepatic RAGE. The present study indicated that the cholinergic anti-inflammatory pathway can significantly inhibit the production of the inflammatory cytokine, S100A8. Following bilateral cervical vagotomy, $\alpha$-BGT administration reversed, while anti-RAGE antibody administration enhanced, the effects of vagus nerve electrical stimulation. Furthermore, the cholinergic anti-inflammatory pathway also inhibited serum AGEs levels and hepatic RAGE protein, which may be associated with the inhibition of S100A8 by vagus nerve electrical stimulation. Therefore, the present study found that the cholinergic anti-inflammatory pathway can inhibit serum S100A8 levels, and serum AGEs and hepatic RAGE protein may mediate the mechanisms underlying the inhibition of S100A8 production and cholinergic anti-inflammatory pathway.

In patients of several acute or chronic inflammatory diseases, S100A8 is increased. Higher concentrations of S100A8 protein has been identified in inflamed tissue of rheumatoid arthritis, compared with healthy individuals (19). Furthermore, elevations of S100A8 protein have been found in a variety of diseases, including acute pancreatitis (20), transplant rejection (21), inflammatory bowel disease (22), myocardial infarction (23) and appendicitis (24). Elevated protein expression of S100A8 has also been described in sepsis and septic shock. Serum S100A8 concentration was increased in patients with severe sepsis (25). The present study also revealed that 
A

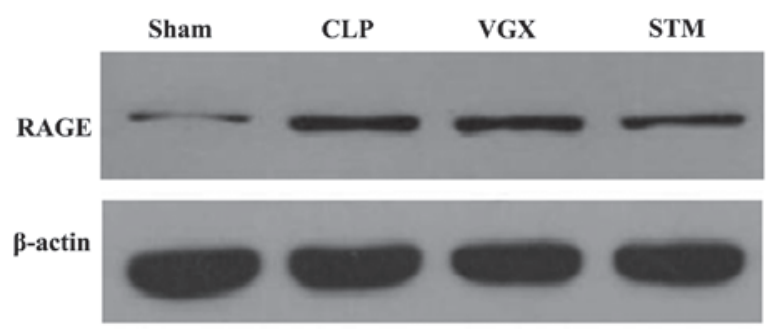

B

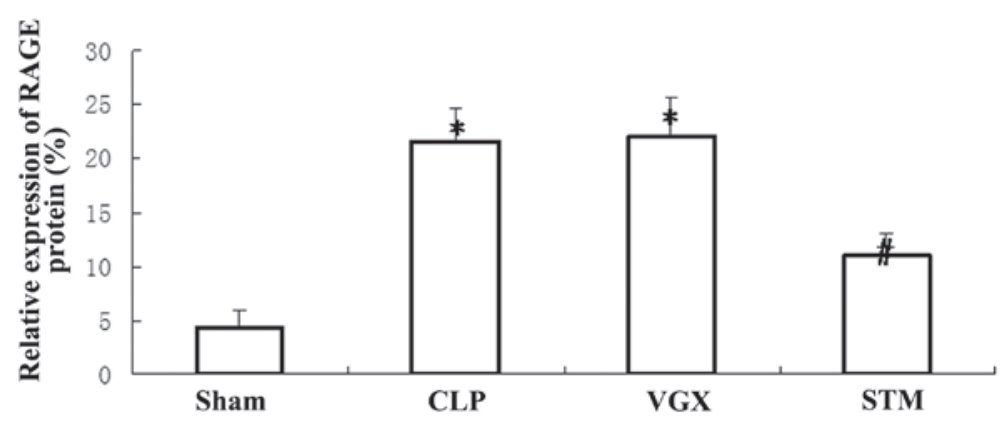

Figure 5. Expression of RAGE protein in different groups at $6 \mathrm{~h}$ after CLP challenge $(\mathrm{n}=6)$. (A) The protein expression of RAGE was determined in the hepatic tissue of Sprague-Dawley rats. Whole cell extracts were immunoblotted with the antibody against RAGE. $\beta$-actin was used as an internal control. The data are a representative figure of six independent experiments. (B) The relative protein expression of RAGE was quantified. The band density was converted into grayscale values followed by normalization against $\beta$-actin. The data are expressed as the mean \pm standard deviation $\left({ }^{*} \mathrm{P}<0.05\right.$, compared with the Sham group; ${ }^{\#} \mathrm{P}<0.05$, compared with the CLP group). CLP, cecal ligation and puncture; VGX, CLP + bilateral cervical vagotomy; STM, CLP + bilateral cervical vagotomy + electrical stimulation; RAGE, receptor for advanced glycation end products.

the S100A8 protein is increased in CLP-induced septic shock rats. Additionally, vagus nerve electrical stimulation reduced the protein expression of S100A8, with attenuation of septic shock. This indicated that the decreased protein expression of S100A8 is associated with the therapeutic effects of vagus nerve electrical stimulation on septic shock, since serum levels of S100A8 protein were decreased during the recovery from septic shock (10).

In the development of multiple organ dysfunction syndrome, induced by septic shock, microvascular blood flow disturbances are pivotal and lead to a decreased perfusion and blood flow velocity, and formation of microthrombi in the liver sinusoids, thereby enhancing liver tissue ischemia and damage (26). In the present study, the serum AST and ALT levels were significantly increased in septic shock, and this indicated that CLP induced the hepatic damage. Vagus nerve electrical stimulation decreased the serum AST and ALT levels, and demonstrated that the cholinergic anti-inflammatory pathway can alleviate the hepatic damage in septic shock rats. Neutrophils are important immune effectors for inflammatory responses, and the recruitment and infiltration of neutrophils are associated with pathology of liver injury induced by a variety of diseases and toxicities (27). In circulating human blood neutrophils, S100A8 constitutes $\sim 20 \%$ of the cytosolic protein fraction (28). S100A8 is an inflammatory factor of neutrophils and has strong chemotactic effects on neutrophils around inflammatory foci. S100A8 can also combine with its partner S100A9 to regulate hepatic CXCL-2 expression and neutrophil recruitment in an injured liver (29). Therefore, in the present study, the elevated S100A8 protein may promote the neutrophil recruitment in injured liver and release of AST and ALT.

In the present study, serum levels of AGEs were significantly increased in septic shock rats, and were decreased by vagus nerve electrical stimulation. AGEs are non-enzymatic glycation/oxidation products of proteins/lipids, and accumulate during natural aging and are augmented in numerous disorders. In septic shock patients, serum levels of AGEs are associated with serum level of asymmetric dimethylarginine, which is significantly higher in septic shock patients compared with in healthy volunteers (30). The accumulation of AGEs is also associated with inflammation, which is confirmed by the report that AGEs increased S100A8 mRNA expression through the RAGE-mitogen-activated protein kinase signaling pathway under diabetic pulp conditions (31). In the present study, the expression pattern of serum AGEs was similar to that of S100A8, therefore, AGEs may be one regulator upstream of S100A8 in septic shock. The present study also suggested that vagus nerve electrical stimulation can inhibit AGEs production. Notably, nicotine has been reported to inhibit the actions of two subtypes of AGEs, AGE-2 and AGE-3, in human monocytes (32). Nicotine is a specific activator of $\alpha 7-n A C h R$ and is reported to inhibit the activation of monocytes. $\alpha 7-n A C h R$ is the receptor for acetylcholine, a neurotransmitter released from the vagus nerve. In the present study, it remains unknown whether vagus nerve electrical stimulation directly reduces the production of AGEs or if decreased serum AGEs are the result of attenuated septic shock. In the senescent mouse brain, declined expression of muscarinic acetylcholine receptor M1 (mAChR1), another receptor subtype for acetylcholine, is correlated with increased expression of AGEs (33). Therefore, the regulation between the vagus nerve and AGEs deserves further investigation.

RAGE can activate multiple inflammatory pathways of numerous physiological and pathological processes. Recently, RAGE has been observed to serve important roles in sepsis by perpetuating inflammation (34). In the present 
study, the protein expression of hepatic RAGE was significantly increased in septic shock rats, and was decreased by vagus nerve electrical stimulation. RAGE is a transmembrane receptor of the immunoglobulin superfamily. Deletion of RAGE inhibits the lethal effects of septic shock, induced by CLP (35), and this indicates that RAGE may serve a pro-inflammatory role and promote the development of septic shock. Notably, compared with healthy volunteers, septic patients exhibited elevated plasma soluble RAGE (sRAGE) concentrations, and among septic patients, non-survivors had higher plasma sRAGE concentrations compared with the survivors, indicating that sRAGE may be a parameter for severity and outcome of septic patients (36). Up until now, no report about the regulation between the vagus nerve and RAGE has been published. Therefore, it remains unknown whether vagus nerve electrical stimulation directly reduces the protein expression of hepatic RAGE or if decreased RAGE protein is the result of attenuated septic shock. It was reported that AGEs upregulate the expression of RAGE in various tissue types, and form a positive feedback loop (37). The positive association between serum AGEs and serum sRAGE was observed in non-diabetic subjects and patients with type 2 diabetes $(38,39)$. Therefore, hepatic RAGE protein may be regulated by AGEs in septic shock rats. In THP-1 human monocytes, AGE pre-incubation resulted in upregulation of RAGE, and AGE pre-treatment, followed by interleukin (IL)- 6 or tumor necrosis factor (TNF) $\alpha$ stimulation further increased the mRNA expression levels of S100A8 and S100A9 and increased the release of S100A8/A9 into the cell culture supernatant. AGEs-mediated S100A8/A9 expression and release were RAGE-dependent (40). Therefore, the hepatic RAGE protein may be regulated by AGEs and RAGE protein also may be a regulator upstream of S100A8 in septic shock rats. Furthermore, since S100A8/A9 has been identified as a ligand of RAGE (40), the protective effect of S100A8 is mediated by RAGE in septic shock rats. This indicated that inhibition of RAGE has potential therapeutic effect on septic shock. Notably, in the present study, anti-RAGE antibody pre-treatment significantly enhanced MAP elevation and S100A8 decrease induced by vagus nerve electrical stimulation. The present result is in accordance with a previous report that anti-RAGE monoclonal antibody served a protective effect and offered a survival advantage to septic mice (41).

However, the precise mechanisms underlying the inhibitory effects of vagus nerve electrical stimulation on S100A8 and RAGE proteins remains unclear. In addition, the roles of other cytokines, including IL-6 and TNF $\alpha$, require further investigation, including their effect on septic shock and the expression of S100A8.

In conclusion, vagus nerve electrical stimulation attenuated hypotension and liver injury, decreased the production of the pro-inflammatory cytokine, S100A8 in vivo. The vagus nerve released acetylcholine, and may inhibit the release and synthesis of AGEs significantly, which regulate the expression levels of S100A8 and RAGE. The protective effect of S100A8 on septic shock may be mediated by RAGE, as the anti-RAGE antibody can enhanced the protective effects of vagus nerve electrical stimulation. The present study demonstrated that S100A8 may be a diagnostic marker for sepsis and septic shock. Further investigation is required to evaluate the feasibility of S100A8 as a therapeutic target in septic shock.

\section{References}

1. Pravda J: Metabolic theory of septic shock. World J Crit Care Med 3: 45-54, 2014.

2. Schulte W, Bernhagen J and Bucala R: Cytokines in sepsis: Potent immunoregulators and potential therapeutic targets-an updated view. Mediators Inflamm 2013: 165974, 2013.

3. King EG, Bauzá GJ, Mella JR and Remick DG: Pathophysiologic mechanisms in septic shock. Lab Invest 94: 4-12, 2014.

4. Averill MM, Kerkhoff C and Bornfeldt KE: S100A8 and S100A9 in cardiovascular biology and disease. Arterioscler Thromb Vasc Biol 32: 223-229, 2012.

5. Goyette J and Geczy CL: Inflammation-associated S100 proteins: New mechanisms that regulate function. Amino Acids 41: 821-842, 2011.

6. Sohnle PG, Hunter MJ, Hahn B and Chazin WJ: Zinc-reversible antimicrobial activity of recombinant calprotectin (migration inhibitory factor-related proteins 8 and 14). J Infect Dis 182: 1272-1275, 2000.

7. Viemann D, Barczyk K, Vogl T, Fischer U, Sunderkötter C, Schulze-Osthoff K and Roth J: MRP8/MRP14 impairs endothelial integrity and induces a caspase-dependent and -independent cell death program. Blood 109: 2453-2460, 2007.

8. Ryckman C, Vandal K, Rouleau P, Talbot M and Tessier PA: Proinflammatory activities of S100: Proteins S100A8, S100A9, and S100A8/A9 induce neutrophil chemotaxis and adhesion. J Immunol 170: 3233-3242, 2003.

9. Viemann D, Strey A, Janning A, Jurk K, Klimmek K, Vogl T, Hirono K, Ichida F, Foell D, Kehrel B, et al: Myeloid-related proteins 8 and 14 induce a specific inflammatory response in human microvascular endothelial cells. Blood 105: 2955-2962, 2005.

10. Payen D, Lukaszewicz AC, Belikova I, Faivre V, Gelin C, Russwurm S, Launay JM and Sevenet N: Gene profiling in human blood leucocytes during recovery from septic shock. Intensive Care Med 34: 1371-1376, 2008.

11. Martelli D, McKinley MJ and McAllen RM: The cholinergic anti-inflammatory pathway: A critical review. Auton Neurosci 182: 65-69, 2014.

12. Mabley JG, Pacher P and Szabo C: Activation of the cholinergic antiinflammatory pathway reduces ricin-induced mortality and organ failure in mice. Mol Med 15: 166-172, 2009.

13. Johnston GR and Webster NR: Cytokines and the immunomodulatory function of the vagus nerve. Br J Anaesth 102: 453-462, 2009.

14. Tracey KJ: Physiology and immunology of the cholinergic antiinflammatory pathway. J Clin Invest 117: 289-296, 2007.

15. Song XM, Li JG, Wang YL, Hu ZF, Zhou Q, Du ZH and Jia BH: The protective effect of the cholinergic anti-inflammatory pathway against septic shock in rats. Shock 30: 468-472, 2008.

16. Wilson J, Higgins D, Hutting H, Serkova N, Baird C, Khailova L, Queensland K, Vu Tran Z, Weitzel L and Wischmeyer PE: Early propranolol treatment induces lung heme-oxygenase-1, attenuates metabolic dysfunction and improves survival following experimental sepsis. Crit Care 17: R195, 2013.

17. Singleton KD and Wischmeyer PE: Distance of cecum ligated influences mortality, tumor necrosis factor-alpha and interleukin-6 expression following cecal ligation and puncture in the rat. Eur Surg Res 35: 486-491, 2003.

18. Bonaz B, Picq C, Sinniger V, Mayol JF and Clarençon D. Vagus nerve stimulation: From epilepsy to the cholinergic anti-inflammatory pathway. Neurogastroenterol Motil 25: 208-221, 2013.

19. Odink K, Cerletti N, Brüggen J, Clerc RG, Tarcsay L, Zwadlo G, Gerhards G, Schlegel R and Sorg C: Two calcium-binding proteins in infiltrate macrophages of rheumatoid arthritis. Nature 330: 80-82, 1987.

20. Farkas G Jr, Tiszlavicz Z, Takács T, Szabolcs A, Farkas G, Somogyvári F and Mándi Y: Analysis of plasma levels and polymorphisms of S100A8/9 and S100A12 in patients with acute pancreatitis. Pancreas 43: 485-487, 2014.

21. Ikemoto M, Matsumoto S, Egawa H, Okitsu T, Iwanaga $Y$, Umemoto S, Itoh $\mathrm{H}$, Murayama $\mathrm{H}$ and Fujita $\mathrm{M}$ : A case with transient increases in serum S100A8/A9 levels implying acute inflammatory responses after pancreatic islet transplantation. Ann Clin Biochem 44: 570-572, 2007. 
22. Leach ST, Yang Z, Messina I, Song C, Geczy CL, Cunningham AM and Day AS: Serum and mucosal S100 proteins, calprotectin (S100A8/S100A9) and S100A12, are elevated at diagnosis in children with inflammatory bowel disease. Scand J Gastroenterol 42: 1321-1331, 2007.

23. Katashima T, Naruko T, Terasaki F, Fujita M, Otsuka K, Murakami S, Sato A, Hiroe M, Ikura Y, Ueda M, et al: Enhanced expression of the S100A8/A9 complex in acute myocardial infarction patients. Circ J 74: 741-748, 2010.

24. Bealer JF and Colgin M: S100A8/A9: A potential new diagnostic aid for acute appendicitis. Acad Emerg Med 17: 333-336, 2010.

25. van Zoelen MA, Vogl T, Foell D, Van Veen SQ, van Till JW, Florquin S, Tanck MW, Wittebole X, Laterre PF, Boermeester MA, et al: Expression and role of myeloid-related protein-14 in clinical and experimental sepsis. Am J Respir Crit Care Med 180: 1098-1106, 2009.

26. Spapen H: Liver perfusion in sepsis, septic shock and multiorgan failure. Anat Rec (Hoboken) 291: 714-720, 2008

27. Ramaiah SK and Jaeschke H: Role of neutrophils in the pathogenesis of acute inflammatory liver injury. Toxicol Pathol 35: 757-766, 2007.

28. Edgeworth J, Gorman M, Bennett R, Freemont P and Hogg N Identification of p8,14 as a highly abundant heterodimeric calcium binding protein complex of myeloid cells. J Biol Chem 266: 7706-7713, 1991.

29. Moles A, Murphy L, Wilson CL, Chakraborty JB, Fox C, Park EJ, Mann J, Oakley F, Howarth R, Brain J, et al: A TLR2/S100A9/CXCL-2 signaling network is necessary for neutrophil recruitment in acute and chronic liver injury in the mouse. J Hepatol 60: 782-791, 2014.

30. Nakamura T, Sato E, Fujiwara N, Kawagoe Y, Suzuki T, Ueda Y, Yamada S, Shoji H, Takeuchi M,Ueda S, et al: Circulating levels of advanced glycation end products (AGE) and interleukin-6 (IL-6) are independent determinants of serum asymmetric dimethylarginine (ADMA) levels in patients with septic shock. Pharmacol Res 60: 515-518, 2009.

31. Nakajima Y,Inagaki Y,Kido J and Nagata T: Advanced glycation end products increase expression of S100A8 and A9 via RAGE-MAPK in rat dental pulp cells. Oral Dis 21: 328-334, 2015.

32. Takahashi HK, Liu K, Wake H, Mori S, Zhang J, Liu R, Yoshino $T$ and Nishibori $M$ : Effect of nicotine on advanced glycation end product-induced immune response in human monocytes. J Pharmacol Exp Ther 332: 1013-1021, 2010.
33. Zhang X, Jin C, Li Y, Guan S, Han F and Zhang S: Catalpol improves cholinergic function and reduces inflammatory cytokines in the senescent mice induced by D-galactose. Food Chem Toxicol 58: 50-55, 2013.

34. Bopp C, Bierhaus A, Hofer S, Bouchon A, Nawroth PP, Martin E and Weigand MA: Bench-to-bedside review: The inflammation-perpetuating pattern-recognition receptor RAGE as a therapeutic target in sepsis. Crit Care 12: 201, 2008.

35. Liliensiek B, Weigand MA, Bierhaus A, Nicklas W, Kasper M, Hofer S, Plachky J, Gröne HJ, Kurschus FC, Schmidt AM, et al: Receptor for advanced glycation end products (RAGE) regulates sepsis but not the adaptive immune response. J Clin Invest 113: $1641-1650,2004$

36. Bopp C, Hofer S, Weitz J, Bierhaus A, Nawroth PP, Martin E, Büchler MW and Weigand MA: sRAGE is elevated in septic patients and associated with patients outcome. J Surg Res 147: 79-83, 2008.

37. Yamagishi S, Nakamura $\mathrm{K}$ and Matsui T: Advanced glycation end products (AGEs) and their receptor (RAGE) system in diabetic retinopathy. Curr Drug Discov Technol 3: 83-88, 2006.

38. Yamagishi S, Adachi H, Nakamura K, Matsui T, Jinnouchi Y, Takenaka K, Takeuchi M, Enomoto M, Furuki K, Hino A, et al: Positive association between serum levels of advanced glycation end products and the soluble form of receptor for advanced glycation end products in nondiabetic subjects. Metabolism 55: 1227-1231, 2006

39. Nakamura K, Yamagishi S, Adachi H, Matsui T, Kurita-Nakamura Y, Takeuchi M, Inoue H and Imaizumi T: Serum levels of soluble form of receptor for advanced glycation end products (sRAGE) are positively associated with circulating AGEs and soluble form of VCAM-1 in patients with type 2 diabetes. Microvasc Res 76: 52-56, 2008.

40. Eggers K, Sikora K, Lorenz M, Taubert T, Moobed M, Baumann G, Stangl K and Stangl V: RAGE-dependent regulation of calcium-binding proteins S100A8 and S100A9 in human THP-1. Exp Clin Endocrinol Diabetes 119: 353-357, 2011.

41. Christaki E, Opal SM, Keith JC Jr, Kessimian N, Palardy JE, Parejo NA, Tan XY, Piche-Nicholas N, Tchistiakova L, Vlasuk GP, et al: A monoclonal antibody against RAGE alters gene expression and is protective in experimental models of sepsis and pneumococcal pneumonia. Shock 35: 492-498, 2011. 